\section{Behandlung der Virushepatitiden - Revolution in 50 Jahren}

\author{
M. Manns ${ }^{1}$ \\ ${ }^{1}$ Klinik für Gastroenterologie, Hepatologie und Endokrinologie, \\ Medizinische Hochschule Hannover (MHH), Hannover, Helm- \\ holtz-Zentrum für Infektionsforschung (HZI), Braunschweig
}

Am Anfang stand die Entdeckung des sogenannten AustraliaAntigens im Serum im Jahre 1965, welches sich als Hüllprotein des Hepatitis-B-Virus (HBV) herausstellte und als Basis des HBV-Impfstoffes diente, der 1981 zugelassen wurde. Die anschließende Entdeckung des Hepatitis-B-Virus selbst wurde gefolgt von der Entdeckung des Hepatitis-A (HAV), -D (HDV), -E (HEV) und schließlich zuletzt des Hepatitis-C-Virus (HCV) im Jahre 1989. Die Entdeckung der fünf verschiedenen Hepatitis-Viren wurde gefolgt von Impfstoffen gegen HBV, HAV und HEV und Therapien für die HBV, HDV und vor allem HCV. Diese drei Hepatitisvirusinfektionen können chronisch werden und verursachen somit chronische Hepatitis, Leberzirrhose und Leberzellkarzinom. Die Therapie der viralen Hepatitiden $B$ und $C$ hat sich in den letzten 30 Jahren kontinuierlich weiterentwickelt und erfährt aktuell nach der Einführung Interferon-freier Therapien für die Hepatitis C eine Revolution. Durch sie wurde erstmals eine chronische Virusinfektion des Menschen heilbar. Die wesentlichen Entwicklungen in der Therapie der Virushepatitiden werden zusammengefasst, deren Basis die Entdeckung der einzelnen Viren und anschließend die Aufschlüsselung des jeweiligen Lebenszyklus war.

\section{Hepatitis A}

Die Hepatitis A ist eine akute, in der Regel selbstlimitierte Erkrankung, die nicht spezifisch antiviral behandelt wird. Sollte es zum akuten Leberversagen kommen, erfolgt die Behandlung wie beim Leberversagen anderer Genese. Es gibt eine effektive aktive Impfung gegen Hepatitis A, die als Indikationsimpfung bei Risikopersonen und bei Reisenden in Risikogebiete, z.B. tropische Länder, eingesetzt wird.

\section{Hepatitis B}

Während die HBV-Impfung seit 1981 verfügbar ist, hat die Therapie der Hepatitis B erst in den neunziger Jahren des letzten Jahrhunderts ihren Durchbruch erlebt. Seit der Zulassung des nukleotidischen Polymeraseinhibitors Tenofovir für Hepatitis B im Jahre 2008 wurde kein neues Medikament mehr zur Behandlung der Hepatitis B zugelassen.

In den letzten Jahren wurden gut etablierte Leitlinien zur Behandlung der Hepatitis B publiziert, die auch weiterhin Gültigkeit besitzen (DGVS: Cornberg et al., Z. Gastroenterol 2011; EASL: J of Hepatology 2012). Grundsätzlich werden zwei verschiedene Behandlungskonzepte unterschieden: Zum einen kann durch potente HBV-Polymeraseinhibitoren eine effektive Hemmung der Virusreplikation erreicht werden, wobei die Resistenzentwicklung mit den hochpotenten Substanzen Entecavir und Tenofovir praktisch kein Problem mehr darstellt. Allerdings benötigen die meisten Patienten aktuell eine lebenslange Therapie; eine Beendigung der Nukleos(t)id- analogatherapie ist derzeit nur in Ausnahmefällen möglich. In mehreren Studien konnte gezeigt worden, dass eine erfolgreiche Langzeittherapie der Hepatitis B mit einer Rückbildung von frühen Leberzirrhosen und einer Reduktion der Bildung eines hepatozellulären Karzinoms assoziiert ist. Weiterhin kann eine Therapie mit pegyliertem Interferon-alpha (pegIFN-alpha) für ein Jahr in Erwägung gezogen werden. Damit ist eine zeitlich limitierte Therapie möglich; zudem ist bei einigen Patienten nicht nur eine HBV-DNA-Reduktion, sondern sogar ein Verlust des HBsAg ein realistischer klinischer Endpunkt, was einer serologischen und auch klinischen Heilung entspricht. Zukünftige Herausforderungen betreffen die Entwicklung einer personalisierten Therapie der chronischen Hepatitis B durch die Kombination beider Therapiekonzepte. Als eine vielversprechende Methode zur Individualisierung der Behandlungsdauer wird die quantitative HBsAgMessung diskutiert; weitere Biomarker werden entwickelt. Hoffnungsvolle innovative Medikamente zur Stimulation der angeborenen Immunität (,innate immunity“) wie z.B. TollLike Rezeptor Agonisten (TLR-7) und der erworbenen Immunität („adaptive immunity“) wie z.B. therapeutische Impfstoffe oder auch Inhibitoren der Hepatitis-B-Aufnahme in die Leberzelle sind in frühen Phasen der klinischen Entwicklung wie auch Nucleocapsidinhibitoren. Insgesamt erlebt die Hepatitis-B-Therapie wieder zunehmendes Interesse mit zahlreichen neuen Therapieansätzen in präklinischer und früher klinischer Entwicklung.

\section{Hepatitis C}

Begonnen hat die Therapie der Hepatitis C 1986 vor Entdeckung des Virus, als die Krankheit noch als non A, non B Hepatitis bezeichnet wurde. Rekombinantes Interferon alfa 2 b und natürliches, biochemisch gereinigtes Interferon alpha waren der Beginn mit etwa 10\% Heilung und einer Therapiedauer von 6-12 Monaten. Verbesserungen der Therapie wurden durch Weiterentwicklungen der Diagnostik mit dem Einsatz der quantitativen HCV-RNA-Bestimmung im Serum und der sich anschließenden individuellen Anpassung der Therapiedauer je nach frühzeitigem Abfall der Viruslast ermöglicht. Medikamentöse Innovationen stellten die Entwicklung verzögert wirksamer pegylierter Interferone und deren Kombination mit Ribavirin dar. Zwar konnte somit erstmals eine chronische Viruserkrankung des Menschen geheilt werden, aber die lange Therapiedauer von 6 bis 72 Monaten, die mit dieser Therapie verbundenen signifikanten Nebenwirkungen sowie die vor allem beim häufigsten Genotyp 1 begrenzte Wirksamkeit mit 50\% stellten wesentliche Limitationen dar. Mit der Entwicklung zahlreicher neuer direkt-antiviraler Medikamente (sog. DAAs) ist eine neue Epoche in der Therapie der chronischen Hepatitis $C$ angebrochen. Im Jahre 2011 hatte die Einführung der Triple-Therapie von PEG-Interferon alfa, Ribavirin und einem der beiden Proteaseinhibitoren Telaprevir oder Boceprevir bereits eine erste deutliche Verbesserung der Heilungschancen auf um die $70 \%$ gebracht. Allerdings war die Wirkung dieser Proteaseinhibitoren der ersten Generation an den gleichzeitigen Einsatz von Interferon und Ribavirin gebunden, und diese sind mit einer zum Teil ausgeprägten Toxizität verbunden. Im Januar 2014 ist nun ein hochpotenter nukleotidischer Polymeraseinhibitor (Sofosbuvir) 
gegen $\mathrm{HCV}$ zugelassen worden, der in Kombination mit Ribavirin erstmals eine Interferon-freie Therapie zumindest für die HCV-Genotypen 2 und 3 sowie im Falle einer InterferonKontraindikation bei verlängerter Therapiedauer auch für andere Genotypen ermöglicht. Im Laufe des Jahres 2014 wurden weitere direkt-antivirale Substanzen zugelassen; im Mai 2014 der erste Proteaseinhibitor der 2. Generation, Simeprevir, und im August 2014 der erste NS5A-Inhibitor Daclatasvir, was die therapeutischen Möglichkeiten weiter verbessert hat. Seit Anfang 2015 stehen zwei neue Kombinationstherapien in Deutschland zur Verfügung, die eine Ausheilung der HCVInfektion bei über $90 \%$ der Patienten innerhalb von 3 bis maximal 6 Monaten Therapie ohne Interferone ermöglichen. Es handelt sich einerseits um die Kombination eines HCV-Proteaseinhibitors (Paritaprevir) - geboostert mit Ritonavir mit einem NS5A-Inhibitor (Ombitasvir) sowie einem nichtnukleotidischen Polymeraseinhibitor (Dasabuvir) mit oder ohne Ribavirin. Anderseits wurde im November 2014 die Kombination des nukleotidischen Polymeraseinhibitors Sofosbuvir mit einem NS5A-Inhibitor (Ledipasvir) in einer Tablette als Fixed Dose Combination (FDC) zugelassen. Mit dieser FDC aus SOF/LDV ist bei bestimmten Voraussetzungen auch schon eine Verkürzung der Therapie auf 8 Wochen möglich. In Japan und weiteren Ländern Asiens wurde für den dort überwiegend vorkommenden Genotyp 1b die Kombination von Daclatasvir mit dem Proteaseinhibitor Asunaprevir als weitere Interferon-freie Therapie zugelassen. Im Jahre 2016 kamen dann zwei weitere Therapien zur Zulassung. Zunächst Sofosbuvir in Kombination mit dem NS5A-Inhibitor Velpatasvir. Diese Kombination erzielt bei allen Genotypen hohe Heilungsraten, so auch bei dem bis dato schwer zu behandelnden Genotyp 3. Als weiteres kam für die Genotypen 1 und 4 Elbatasvir plus Grazoprevir hinzu; letzteres ist auch bei Patienten mit Niereninsuffizienz anwendbar. Somit stehen für alle Genotypen und Patientengruppen gut verträgliche Interferonfreie Therapien zur Verfügung, die Heilungsraten von 95$100 \%$ erreichen. Die Hepatitis-C-Therapie erfährt somit einen dramatischen Umbruch. Es lohnt sich nun im besonderen, HCV-Screening-Programme zu etablieren und Patienten mit bekannter Hepatitis $C$ einer Therapie zuzuführen, da nur so mittel- bis langfristig die HCV-assoziierte Morbidität und Mortalität signifikant gesenkt werden kann.

\section{Hepatitis D}

Die chronische Hepatitis D gilt als die schwerste Form der chronischen Virushepatitis, da im Verlauf ein besonders hohes Risiko für Leberzirrhose und Leberzellkarzinome zu verzeichnen ist, und Leberzirrhosen durch HDV Infektion im Schnitt 10 Jahre früher auftreten als bei chronischer Hepatitis B. Die Hepatitis D kann nur als Koinfektion mit der Hepatitis B auftreten, es handelt sich somit immer um eine Doppelinfektion von HBV und HDV. Die einzige bisher wirksame Therapieoption ist Peg-Interferon alfa. Eine große Studie des Kompetenznetzes Hepatitis hatte zeigen können, dass etwa 25\% der Patienten von einer Therapie von 12 Monaten profitieren.
Längere Therapiedauer sowie Kombinationstherapien mit HBV-Nukleotid- oder Nukleosidinhibitoren wie Adefovir und Tenofovir scheinen keinen zusätzlichen Nutzen zu haben. Zurzeit sind mehrere neue Substanzen in der präklinischen und klinischen Entwicklung wie der Prenylationshemmer Lorafenib oder der HBV Entry Inhibitor Myrcludex.

\section{Hepatitis E}

HEV-Infektionen sind in Deutschland wahrscheinlich sehr viel häufiger als bisher angenommen, da zoonotische Übertragungen des Genotyps 3 zum Beispiel durch Verzehr von nicht ausreichend erhitztem Schweinfleisch möglich sind. Während bei immunkompetenten Personen eine HEV-Infektion in der Regel zur klinisch unauffälligen Serokonversion oder zu einer akuten, selbstlimitierten Hepatitis führt, wurden in den letzten Jahren zahlreiche Fälle einer chronischen HEV-Infektion, assoziiert mit progressiver Lebererkrankung, bei verschiedenen Kohorten von immunsupprimierten Personen wie zum Beispiel Organtransplantierten beschrieben. Eine Ribavirin-Monotherapie ist effektiv und sollte zwischen 3 und 5 Monaten durchgeführt werden. Vor 2 Jahren wurde in China ein Impfstoff gegen Hepatitis E zugelassen, welcher allerdings in Europa bisher nicht verfügbar ist. HEV-Infektionen sind ganz aktuell auch mit neurologischen Symptomen und dem Auftreten von Guillain-Barré-Syndromen assoziiert worden.

Interessenkonflikte: Forschungsförderung: Roche, BristolMyers Squibb, Gilead, Boehringer Ingelheim, Novartis, Merck (MSD), Janssen, GlaxoSmithKline, Biotest. Beratergebühr/Honorar: Roche, Bristol-Myers Squibb, Gilead, Boehringer Ingelheim, Novartis, Merck (MSD), Janssen, Idenix, GlaxoSmithKline, Biotest, Achillion. Reisekostenunterstützung: Roche, Bristol-Myers Squibb, Gilead, Boehringer Ingelheim, Merck (MSD), Janssen, GlaxoSmithKline, Biotest. Aufsichtsratstätigkeit: Bristol-Myers Squibb, Gilead, Boehringer Ingelheim, Novartis, Merck (MSD), Janssen. Vortragshonorar: Roche, Bristol-Myers Squibb, Gilead, Boehringer Ingelheim, Merck (MSD), Janssen.

\section{Bibliografie}

DOI http://dx.doi.org/10.1055/s-0042-114458

Drug Res 2016; 66, Suppl. 1: S13-S14

(c) Georg Thieme Verlag KG Stuttgart · New York .

ISSN 2194-9379

\section{Korrespondenzadresse}

Univ.-Prof. Dr. Michael P. Manns

Direktor der Klinik für Gastroenterologie, Hepatologie u. Endokrinologie

Medizinische Hochschule Hannover

Carl-Neuberg-Straße 1

30625 Hannover

manns.michael@mh-hannover.de 